\title{
EDITORIAL
}

\section{Introduction to a Supplement on Innovative Approaches to Studying Health Outcomes in Rare Diseases}

\author{
Aaron S. Kesselheim, M.D., J.D., M.P.H and Joshua J. Gagne, Pharm.D., Sc.D.
}

From the Program On Regulation, Therapeutics, And Law, Division of Pharmacoepidemiology and Pharmacoeconomics, Department of Medicine, Brigham and Women's Hospital and Harvard Medical School, Boston, MA, USA.

J Gen Intern Med 29(Suppl 3):S709-11

DOI: $10.1007 / \mathrm{s} 11606-014-2921-\mathrm{y}$

(c) Society of General Internal Medicine 2014

$\mathrm{O}$ ver the past decade, rare diseases have assumed an increasingly prominent place in health care discourse in the US and around the world. Rare diseases currently comprise approximately 6,000 different disease types, affecting about $6-8 \%$ of people, ${ }^{1}$ or about the same proportion of the US population with diabetes. ${ }^{2}$ However, the number of rare diseases continues to rise, as new genomic data have led physicians and scientists to recognize that many diseases thought to be single entities may actually be made up of numerous genetically unique rare subtypes that have different responses to therapy and varying prognoses. In addition, drugs for rare diseases make up the largest growing category of new molecular entities approved by regulators in the US and EU. ${ }^{3,4}$ New legislative programs in the US and EU have sought to encourage greater investment in research for certain types of rare diseases, including neglected tropical diseases. ${ }^{5}$ At the same time, investment by charitable foundations and patient interest groups related to rare diseases has led to some notable therapeutic advances, such as ivacaftor, a breakthrough therapy for a rare subtype of cystic fibrosis. ${ }^{6}$

Despite these advances, there remains an urgent need for research into the biological basis and natural history of many rare diseases, the health outcomes that patients with rare diseases might expect, and how various clinical interventions might affect those outcomes. Emily Kramer-Golinkoff provides powerful first-hand insights into the urgency faced by rare disease patients in her "Healing Arts" essay in this issue. However, there are many obstacles to conducting such research. Studies of rare diseases not only often include few patients, limiting statistical study power, but because a large proportion of patients with the same rare disease may be included in a particular study, there may be limited opportunity to replicate study findings in external populations. The geographic spread of patients who might qualify for a prospective study complicates recruitment and follow-up and increases study costs. In trials of medical interventions,

Published online July 17, 2014 patients sometimes opt not to enroll because of concerns that they would receive a placebo rather than an active treatment. Some patients also seek and gain access to drugs outside of trials, further limiting recruitment and enrollment.

These challenges necessitate innovative approaches to research, which was a key motivation for producing this journal supplement. The articles herein focus on novel ways of evaluating patient health outcomes in rare diseases. We identified four general facets of research for rare diseases that necessitate such innovative approaches:

(1) Rare disease patients' health outcomes in clinical trials. Rigorous approaches to randomized trials in rare diseases are needed. In a recent study, we found that cancer drugs for rare diseases were more likely than non-orphan cancer drugs to be approved on the basis of methodologically weaker assessments. That is, these trials were more likely to be nonrandomized, unblinded, single-arm trials and more likely to consider only intermediate endpoints such as "disease response" rather than survival. ${ }^{7}$

Therefore, in organizing this supplement, we sought to include manuscripts describing creative solutions to improve the conduct of randomized trials for the development and testing of rare disease treatment, other tools or approaches that might be applied to address the unique features of studying treatments in rare diseases, and the implications of these nontraditional clinical trial methods for rare diseases patients. In this issue, Retsch-Bogart et al. identify clinical research metrics to improve clinical trial performance in the context of cystic fibrosis, showing that certain characteristics of clinical trials involving rare diseases are directly related to the success of the trial in attracting patients.

(2) Emerging data sources and novel analytic methods for observational research on health outcomes among patients with rare diseases. Due in part to the challenges of conducting the randomized trials described above, observational studies are taking on a more prominent role in evidence generation for patients with rare diseases. Yet, in a previous review of methods proposed for or used to study health outcomes in rare diseases, we found very little 
innovation in the area of observational research methods, even with the increases in available data resources. Superb work has been done recently by rare disease patients, their advocacy groups, and their physicians to build registries of important health outcomes among rare disease patients. ${ }^{8}$ In addition, many existing and nascent networks of electronic healthcare databases bring together data on large numbers of patients that could be used to study outcomes in rare diseases.

In this issue, we feature several articles that address both processes of data aggregation and building federated data networks to amass larger patient populations with increasingly rich clinical information and methods for analyzing data in such environments. Nigwekar et al. and Royer et al. focus on innovative uses of administrative data sources in studying rare diseases. Nigwekar and colleagues developed an algorithm using natural language processing and review of medical and pathology records in electronic healthcare data to identify patients with calciphylaxis and used it to conduct the first national epidemiologic evaluation of this rare disorder in the US. Royer and colleagues describe the process they used to identify patients with fragile $\mathrm{X}$ syndrome, spina bifida, and muscular dystrophies in a novel statewide database in South Carolina that includes demographic and medical information linked to employment, education, disability, and socioeconomic data.

Similarly, Krischer et al. describe the Rare Diseases Clinical Research Network, which has supported 128 studies since 2003, while Thompson et al. present experience from RD-Connect, an initiative that is developing a platform to integrate rare disease registry data, genomic data, biobank data, and bioinformatics tools into a centralized data repository for rare disease research. Maro et al. describe an approach to sequential analysis of drug effects in distributed data networks as a way to understand potential risks of new orphan drugs as quickly as possible after approval.

(3) Integrating stakeholder involvement in assessing health outcomes among patients with rare diseases. Involvement of patients and their caregivers in the research process offers the promise of identifying and prioritizing for study those outcomes of greatest interest to those affected by rare diseases. Engaging such stakeholders can generate patient-centered evidence that helps patients make decisions that reflect their preferred health outcomes. Three articles in this supplement focus on patient-centered health outcomes in the pre- and post-market settings. Thyen et al. assess satisfaction with care in patients with disorders of sex development and identify factors associated with satisfaction, pointing to important gaps in care coordination that can negatively affect outcomes most important to patients. Poon et al. describe an approach to evaluating longitudinal changes in health-related quality of life and for disentangling static and dynamic factors that may affect such changes. Forsythe et al., writing from the PatientCentered Outcomes Research Institute, review evidence about stakeholder engagement in research on rare diseases and highlight successful examples of studies in which patients and stakeholders were meaningfully involved in the research process.

(4) Economic, regulatory, ethical, legal, and social issues related to the study of rare disease health outcomes. Finally, our authors raise a number of related economic, ethical, legal, and social policy questions. For example, how can success in improving some outcomes (as with cystic fibrosis and some rare oncologic conditions) be duplicated in other rare disease fields, with limited financial resources for infrastructure needs such as the coordination of geographically dispersed patients? Do the high costs of orphan drugs limit their impact on patient health outcomes? How should we manage informed consent or other ethical implications of recruiting rare disease patients into innovative clinical trial designs with which researchers have limited experience? Can the current barriers to rare disease research be addressed through crowdsourcing, telemedicine, or other emerging social media tools?

To address these questions, Abrahamyan et al. apply value-of-information methods to prioritizing reimbursement for expensive rare disease treatments. Winquist et al. evaluate a different framework for health policy decision-making around investment in rare diseases that involves reviewing clinical effectiveness, costs, and budget impacts. Anne Pariser and William Gahl target areas of translational science to advance rare disease research and enhance the efficiency of orphan drug development, and Ethan Basch and Antonia Bennett appeal to the immediacy of integrating patientreported outcomes into rare disease research.

The goal of this issue is to illuminate some of the important work being done around the world to improve methods for studying rare diseases. In addition, clinicians may be motivated to consider how they can support the methods described in these pages. For example, internists working with rare disease patients may choose to take a more active role in coordinating patient care, promote collection of unique patient-reported outcomes, or 
provide more robust clinical data on their patients to insurers or institutional data repositories that can support observational research. Finally, it is our hope that this remarkable collection of articles will not only provide insights into some of these novel methods that readers can bring back to their own research, but also spark creative thinking to further enhance methodological innovation in this area and ultimately improve health outcomes for those living with rare diseases.

Corresponding Author: Aaron S. Kesselheim, M.D., J.D., M.P.H; From the Program On Regulation, Therapeutics, And Law, Division of Pharmacoepidemiology and Pharmacoeconomics, Department of MedicineBrigham and Women's Hospital and Harvard Medical School, Boston, MA, USA (e-mail: akesselheim@partners.org).

\section{REFERENCES}

1. Coté T, Kelkar A, Xu K, Braun MM, Phillips MI. Orphan products: an emerging trend in drug approvals. Nat Rev Drug Discov. 2010;9(1):84.

2. NIDDK. National diabetes statistics. 2011. Available at: http:// diabetes.niddk.nih.gov/dm/pubs/statistics/.

3. Kesselheim AS. Innovation and the Orphan Drug Act, 1983-2009: the regulatory and clinical characteristics of approved orphan drugs. In: Field MJ, Boat TF, eds. Accelerating Rare Diseases Research and Orphan Product Development. Washington, DC: National Academy Press; 2010.

4. Joppi R, Bertele' V, Garattini S. Orphan drugs, orphan diseases. The first decade of orphan drug legislation in the EU. Eur J Clin Pharmacol. 2013;69(4):1009-24.

5. Kesselheim AS. An empirical review of major legislation affecting drug development: past experiences, effects, and unintended consequences. Milbank Q. 2011;89(3):450-502.

6. Clancy JP, Jain M. Personalized medicine in cystic fibrosis: dawning of a new era. Am J Respir Crit Care Med. 2012;186(7):593-7.

7. Kesselheim AS, Myers JA, Avorn J. Characteristics of clinical trials to support approval of orphan vs nonorphan drugs for cancer. JAMA. 2011;305:2320-6.

8. European Platform for Patients' Organisations, Science and Industry. Workshop on patients' registries for rare disorders: need for data collection to increase knowledge on rare disorders and optimize disease management and care. 2009. Available: http://www.epposi.org/images/stories/ publications/rarediseases/2009/Registries2009final.pdf. 